\title{
A Survey on People's Impressions of the Paralympic Games
}

\author{
Motomu Haishima, Norihito Kajita, Koichiro Aoki, Chieko Kato \\ Graduate School of Information Sciences and Arts, Toyo University, Saitama, Japan \\ Email: motomuh110@gmail.com
}

How to cite this paper: Haishima, M., Kajita, N., Aoki, K., \& Kato, C. (2020). A Survey on People's Impressions of the Paralympic Games. Open Journal of Social Sciences, 8, 436-444.

https://doi.org/10.4236/jss.2020.84031

Received: March 23, 2020

Accepted: April 20, 2020

Published: April 23, 2020

Copyright (อ 2020 by author(s) and Scientific Research Publishing Inc. This work is licensed under the Creative Commons Attribution International License (CC BY 4.0).

http://creativecommons.org/licenses/by/4.0/

(c) (i) Open Access

\begin{abstract}
In Japan, people's interest in the Paralympics is at a lower level compared with awareness of the Paralympics. This study conducted a questionnaire survey focusing on people's impressions of the Paralympics and Para-Athletes as background factors of the current situation. A preliminary survey was conducted to make questions about these impressions for a main survey. Participants in the preliminary survey described their impressions of the Paralympics and Para-Athletes and 23 questions for the main survey were made based on these descriptions. Participants in the main survey were asked to answer two questions about their experiences of watching Para-Sports and their interest in the Paralympics, along with 23 questions about their impressions of the Paralympics and Para-Athletes. Factor analysis of the answers to the 23 questions was carried out and three factors were extracted: "Admired Hero", "Self-Disciplined People", and "Athletes Encouraging People". In addition, the participants were classified into two groups depending on their experiences of watching Para-Sports and interest in the Paralympics, and each factor score was compared among groups. The results showed there were significant differences in the scores of the second factor, "Self-Disciplined People" between the groups. The second factor was composed of the impressions related to athletes' traits required in their competition life and represented their attitude in detail. This result suggested that people who have watched Para-Sports or who have interest in the Paralympics tend to focus on Para-Athletes' attitudes as athletes, and to be impressed with them.
\end{abstract}

\section{Keywords}

Paralympics, Impression, Questionnaire, Factor Analysis

\section{Introduction}

In Japan, coverage of the Paralympics through various media has been increas- 
ing ahead of the 2020 Tokyo Olympic and Paralympic Games. One of the reasons might be that awareness of the Paralympics has been rising.

According to a survey by Fujita (2016), many respondents knew of not only "the Olympics (98.9\%)" but also "Paralympics (97.3\%)". However, a survey by Sato (2015) showed that only .5 percent of respondents answered correctly to a question about the kinds of disabilities of Paralympic athletes (Para-athletes) although many of the respondents knew of the Paralympics. A survey in 2014 by Yamaha Motor Foundation for Sports (2016) also indicated that the average ratio of respondents who knew of the Para-athletes (3.5\%) was less than the Olympic athletes (40.7\%).

A survey on watching the 2016 Rio Olympic Games Panasonic (2016) reported that 88.2 percent of respondents had watched the Olympic Games on TV. In contrast to this result, according to a survey by Sato (2015), about 49 percent of respondents answered "Yes" to the question "Have you watched the Paralympic Games on TV?". "The Paralympics" is known by Japanese people as well as "the Olympics", however, they do not understand Para-athletes in detail and have less experience watching Paralympic sports (Para-Sports) on TV. Although awareness has been increasing, people's interest in the Paralympics might still not be as high as the Olympics.

The above survey by Fujita (2016) also focused on people's thoughts about Para-athletes and Para-Sports. According to this survey, 35.9 percent of respondents answered "I think so" to the question "People with disabilities are to be pitied," 61.5 percent answered "I think so" to "It is difficult for people with disabilities to live in a similar way to people without disabilities," and 27.5 percent answered "I think so" to "Disabled sports are an unusual sport." These results showed the existence of people who have a negative impression about people with disabilities and disabled sports. The respondents who had experience playing or watching Para-Sports were likely to answer "I do not think so" to the above negative questions. Such experience can lead to a better understanding of people with disabilities and disabled sports.

Considering the results of the previous studies, it is important to increase opportunities for playing and watching disabled sports by spreading information. Before that, to convey a positive image of disabled sports is also important in order to improve people's interest in them. However, the survey by Fujita (2016) focused on the negative image about people with disabilities and disabled sports, and it did not examine the positive image.

The current study conducted a survey on impressions of the Paralympics, which is the most major competition of disabled sports, and the Para-athletes focusing also on positive impressions of them. University students were selected as participants of this study because the previous studies (Fujita, 2016) reported that young people (from 12 to 39 years old) had a relatively positive image about people with disabilities and disabled sports. The university students were included in the above age group of young people and they were considered to be suited to the survey focusing on the positive image of Para-Athletes. The study 
also examined differences of impressions depending on participants' experience watching Para-Sports and interest in the Paralympics. The purpose of this study is to clarify effects of such experience and interest of the participants' impressions.

\section{Preliminary Survey}

A preliminary survey was conducted in June 2017 to create survey questions for a main survey. Participants were 42 students (24 males and 14 females. Four were of unknown gender) of University-A. They were asked to answer a descriptive question about their impressions of the Paralympics and Para-athletes. Based on their answers, the survey questions were created.

\section{Main Survey}

\subsection{Participants}

The main survey was carried out with 66 students (46 males, 20 females) of University-A from October 2017 to January 2018. In this survey, the participants were divided into two groups and their answers were statistically compared between the two groups. Each groups required more than 30 samples for fulfilling the central limit theorem. Because of that, more than 60 people were selected as the participants in the main survey.

\subsection{Method of Survey}

The preliminary survey was conducted asking participants their impressions of the Paralympics and Para-Athletes. The questions (Table 1) were made based on the results of the preliminary survey. The participants in the survey were asked to answer two questions by "Yes" or "No" about their experience watching Para-Sports ("Have you ever seen the Paralympics?") and their interest in the Paralympics ("Are you interested in the Paralympics?"). Furthermore, based on the results of the preliminary survey, 23 survey questions (Table 1) were created about impressions of the Paralympics and Para-Athletes. The participants answered these questions by means of a five-point scale (1: applicable, 2: slightly applicable, 3: neither, 4: slightly not applicable, 5: not applicable).

\subsection{Method of Analysis}

A factor analysis (Varimax rotation, Principal factor method) was conducted using the scores from the 23 questions of participants' impressions. The number of factors was set from three to five. In addition, questions whose factor loadings were less than .4 were removed and the factor analysis was carried out repeatedly until all questions showed the high factor loadings more than .4. In addition, Cronbach's alpha was calculated for each factor in order to verify reliability of the questions.

Based on their responses to the questionnaire, participants were divided into two groups in accordance with the following criteria: "whether the participants 
Table 1. Questions about impressions of the Paralympics and para-athletes.

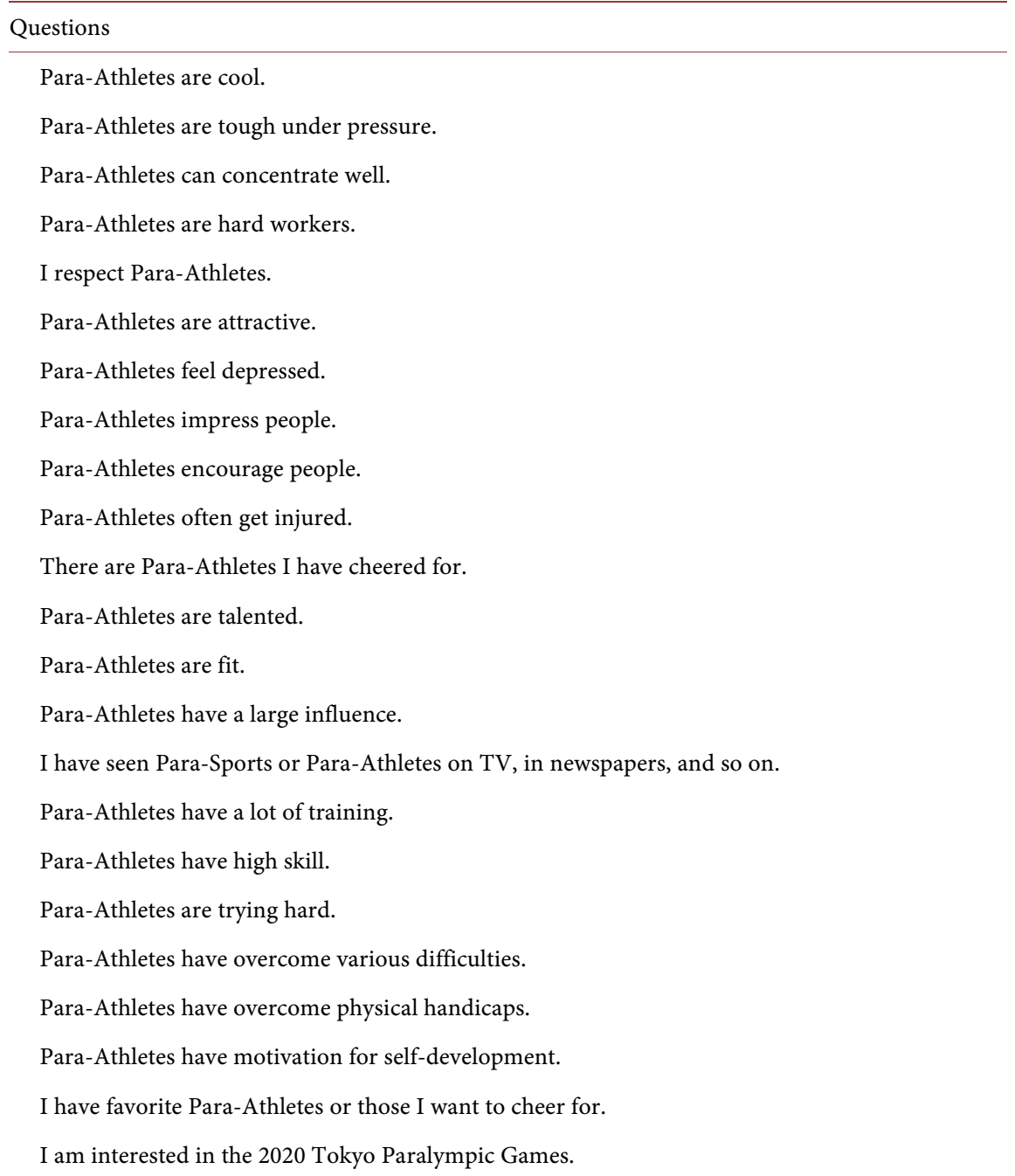

have experience watching the Paralympics" and "whether the participants are interested or not interested in the Paralympics". Furthermore, factor scores calculated by the factor analysis were statistically compared between the two groups depending on the above criteria using $\mathrm{t}$-test.

\section{Results of the Main Survey}

\subsection{Results of the Survey on Impressions}

Twenty-six participants, $40.6 \%$ of the all participants responded "yes" to the question "Have you ever seen the Paralympics?", while thirty-eight participants, $59.4 \%$ of them responded "no". And also, twenty-seven participants, $42.2 \%$ of the all participants responded "yes" to the question "Are you interested in the Paralympics?", while thirty-seven participants, $57.8 \%$ of them responded "no".

\subsection{Results of Factor Analysis and Reliability Analysis}

In the case that the number of factors was set at three, eight questions had high 
factor loadings on the first factor, six questions on the second factor, and six questions on the third factor. The above three factors were adopted because the number of questions, which had high factor loadings on each factor, was well-balanced (Table 2).

The following questions had high factor loadings on the first factor: "Para-Athletes are talented," "There are Para-Athletes I have cheered for," "Para-Athletes are fit," "Para-Athletes are attractive," "I have favorite Para-Athletes or those I want to cheer for," "Para-Athletes have a large influence," "Para-Athletes often get injured," and "I am interested in the 2020 Tokyo Paralympic Games." These questions reflected the participants' feeling that they want to cheer for the Para-Athletes and praise their abilities. Such feelings might be based on the participants' impressions of the Para-Athletes as admired people. Therefore, the first factor was called "Admired Hero". The Cronbach's alpha of the first factor is .85 .

Table 2. Factor loadings of each question.

\begin{tabular}{|c|c|c|c|}
\hline Questions & $\begin{array}{c}\text { First } \\
\text { Factor }\end{array}$ & $\begin{array}{l}\text { Second } \\
\text { Factor }\end{array}$ & $\begin{array}{l}\text { Third } \\
\text { Factor }\end{array}$ \\
\hline Para-Athletes are talented. & .72 & .22 & -.04 \\
\hline There are Para-Athletes I have cheered for. & .72 & .07 & -.08 \\
\hline Para-Athletes are fit. & .70 & .31 & .14 \\
\hline Para-Athletes are attractive. & .68 & .28 & .12 \\
\hline I have favorite Para-Athletes or those I want to cheer for. & .68 & .08 & -.17 \\
\hline Para-Athletes have a large influence. & .56 & .03 & .43 \\
\hline Para-Athletes often get injured. & .56 & -.06 & .30 \\
\hline I am interested in the 2020 Tokyo Paralympic Games. & .42 & .33 & .12 \\
\hline Para-Athletes can concentrate well. & .04 & .87 & .10 \\
\hline Para-Athletes are trying hard. & .05 & .76 & .02 \\
\hline Para-Athletes have high skill. & .03 & .73 & .13 \\
\hline Para-Athletes are tough under pressure. & .29 & .72 & .10 \\
\hline Para-Athletes are cool. & .34 & .49 & .09 \\
\hline Para-Athletes have a lot of training. & .16 & .44 & .23 \\
\hline Para-Athletes feel depressed. & .32 & .44 & .25 \\
\hline Para-Athletes encourage people. & .36 & .06 & .77 \\
\hline Para-Athletes are hard workers. & -.33 & .21 & .58 \\
\hline Para-Athletes impress people. & .31 & .29 & .57 \\
\hline Para-Athletes have overcome various difficulties. & -.13 & .30 & .56 \\
\hline Para-Athletes have overcome physical handicaps. & -.05 & -.04 & .45 \\
\hline I respect Para-Athletes. & .17 & .17 & .40 \\
\hline Contribution Rate & 18.90 & 17.23 & 11.64 \\
\hline Accumulated Contribution Rate & 18.90 & 36.13 & 47.77 \\
\hline
\end{tabular}


The following questions had high factor loadings on the second factor: "Para-Athletes can concentrate well," "Para-Athletes are trying hard," "Para-Athletes have high skill," "Para-Athletes are tough under pressure," "Para-Athletes are cool," "Para-Athletes have a lot of training," and "Para-Athletes feel depressed." These questions were considered to evaluate the Para-Athletes' mental strength and attitude towards competition. Therefore, the second factor was called "Self-Disciplined People". The Cronbach's alpha of the second factor is .83.

The following questions had high factor loadings on the third factor: "Para-Athletes encourage people," "Para-Athletes are hard workers," "Para-Athletes impress people," "Para-Athletes have overcome various difficulties," "Para-Athletes have overcome physical handicaps," and "I respect Para-Athletes." These questions mentioned the psychological influences (e.g., encouraging and impressing people), which the Para-Athletes had on spectators, and the background factors of these influences. Therefore, the third factor was called "Athletes Encouraging People". The Cronbach's alpha of the third factor is .71.

The following two questions, "I have seen Para-Sports or Para-Athletes on $\mathrm{TV}$, in newspapers, and so on" and "Para-Athletes have motivation for self-development," had factor loadings of less than .4 and were removed from the analysis.

\subsection{Results of Comparison Focusing on Experiences of Watching the Para-Sports}

The scores of the first, second, and third factor were compared between the two groups depending on the experiences of watching the Para-Sports by the t-test (Table 3). As a result, there was a significant difference between the scores of the second factor $(t(51.79)=-2.47, p<.05, d=.55)$. The scores of participants who had no experience of watching the Para-Sports were higher than those who had watched Para-Sports.

\subsection{Results of Comparison Focusing on Interest in the Paralympics}

The scores of the first, second, and third factor were compared between the two groups depending on interest in the Paralympics by the t-test (Table 4). As a

Table 3. Each factor scores depending on experiences of watching the para-sports.

\begin{tabular}{cccc}
\hline Factor & Experience & $M(S D)$ & t-value \\
\hline \multirow{2}{*}{ First factor } & Have $(\mathrm{n}=26)$ & $-.20(1.05)$ & -1.44 \\
& Not have $(\mathrm{n}=38)$ & $.14(.84)$ & \\
Second factor & Have $(\mathrm{n}=26)$ & $-.30(.45)$ & $-2.47^{*}$ \\
& Not have $(\mathrm{n}=38)$ & $.20(1.13)$ & .63 \\
Third factor & Have $(\mathrm{n}=26)$ & $.09(1.04)$ & \\
& Not have $(\mathrm{n}=38)$ & $-.06(.85)$ &
\end{tabular}

${ }^{*} p<.05$. 
Table 4. Each factor scores depending on interest in the Paralympics.

\begin{tabular}{cccc}
\hline Factor & Interest & $M(S D)$ & t-value \\
\hline \multirow{2}{*}{ First factor } & Interested $(\mathrm{n}=27)$ & $-.16(.92)$ & -1.15 \\
& Not interested $(\mathrm{n}=37)$ & $.11(.95)$ & \\
\multirow{3}{*}{ Second factor } & Interested $(\mathrm{n}=27)$ & $-.23(.45)$ & $-1.88^{\dagger}$ \\
& Not interested $(\mathrm{n}=37)$ & $.17(1.16)$ & \\
\multirow{2}{*}{ Third factor } & Interested $(\mathrm{n}=27)$ & $-.05(1.00)$ & -.33 \\
& Not interested $(\mathrm{n}=37)$ & $.03(.89)$ & \\
\hline
\end{tabular}

${ }^{\dagger} p<.10$.

result, there was a marginally significant difference between the scores of the second factor $(t(49.39)=-1.88, p<.10, d=.42)$. The scores of participants who had no interest in the Paralympics were higher than those who had interest.

\section{Discussion}

The main study conducted the survey of the impressions of the Paralympics and Para-Athletes. The results of the survey were analyzed statistically.

The three factors that were extracted from the results of the survey on impressions using factor analysis are "Admired Hero", "Self-Disciplined People", and "Athletes Encouraging People". The factor of "Admired Hero" was related to impressions such as "Para-Athletes are talented," "Para-Athletes are attractive" and "Para-Athletes have a large influence," which corresponded to admiration and respect for the Para-Athletes. Compared with the negative impressions in the previous study (Fujita, 2016), the above impressions were not related to whether athletes have disabilities. The participants were considered to have viewpoints recognizing the Para-Athletes positively and purely as athletes.

The factor of "Self-Disciplined People" was related to the impressions that "Para-Athletes can concentrate well," "Para-Athletes are trying hard," "Para-Athletes are tough under pressure," "Para-Athletes are cool" and "Para-Athletes have a lot of training." These impressions are related to the athletes' mental aspects. The self-discipline of the athletes seemed to impress the participants when they went through hard training and overcame pressure in a big match. The participants were presumed to have these impressions focusing on the Para-Athletes' attitudes as athletes.

The factor of "Athletes Encouraging People" was related to the impressions that "Para-Athletes encourage people" and "Para-Athletes impress people" as well as the impressions that "Para-Athletes have overcome various difficulties" and "Para-Athletes have overcome physical handicaps." These impressions related to the athletes' disabilities. Their disabilities are sometimes recognized as "difficulties" or "handicaps" they have to overcome, which can encourage and impress people.

Considering the results of reliability analysis (Cronbach's $\alpha$ of the first fac- 
tor: .85 , the second factor: .83 , and the third factor: .71), the questions seemed to have acceptable reliability.

Furthermore, the three factor scores calculated by the factor analysis were statistically compared between the two groups depending on the participants' experiences of watching the Para-Sports and interest in the Paralympics. As a result, the participants who had no experiences and interest showed a higher score of the second factor, "Self-Disciplined People" than those who had the experiences and interest.

The negative impressions of athletes with disabilities such as "people with disabilities are to be pitied," which were shown in a preliminary study (Fujita, 2016), were considered to pay attention to their disabilities. Therefore, focusing on Para-Athletes' disabilities sometimes leads to their negative impressions.

The third factor, "Athletes Encouraging People" is the image focusing on Para-Athletes' disabilities as the "difficulties" or "handicaps" they have to overcome. In contrast, the first factor, "Admired Hero" and the second factor pay attention to the Para-Athletes' image as top-athletes. In particular, the questions which had high factor loadings on the second factor mainly mentioned athletes' traits required in their competition life. That is, the second factor represents the attitude of athletes engaging in competition in detail. People who have watched Para-Sports or had interest in the Paralympics are considered to be able to specifically imagine competition scenes of these sports and Para-athletes' competition life. Therefore, promoting understanding of disabled sports through watching and participating in Para-Sports can influence people's impression of $\mathrm{Pa}$ ra-Athletes.

\section{Conclusion}

This study conducted a survey of people's impressions of the Paralympic Games and Para-Athletes. The results of the factor analysis clarified the dimensions of the impressions such as the first factor of "Admired Hero", the second factor of "Self-Disciplined People", which was the impression as top-athletes, and the third factor of "Athletes Encouraging People", which was the impression as those having overcome their handicaps.

The factor scores of the above factors were statistically compared between the two groups depending on the participants' experiences of watching the Para-Sports and interest in the Paralympics. The results showed that there were significant or marginally significant differences between the scores of the second factor (Self-Disciplined People) in these two groups. Therefore, promoting watching the Para-Sports and interest in the Paralympics can lead to the improvement of the Para-Athletes' image such as "Self-Disciplined People". People may feel the Para-Athletes' physical and mental strength and their attitudes toward competitions by watching Para-Sports and deepening their understanding of the games and athletes.

Promotional activities for the Paralympics for building the above impression 
("Self-Disciplined People") of the Para-Athletes can be useful for improvement of an overall impression of Para-Sports. Furthermore, it is necessary to identify factors related to building impressions of the athletes in order to establish positive impressions such as "Admired Hero" and "Athletes Encouraging People". Clarification of factors improving these impressions is required through practical activities such as experiential sessions of Para-Sports in the future.

The results of this study can be utilized for promotion activities of Para-Sports. In particular, this study showed that the second factor ("Self-Disciplined People") of impressions of the Paralympics and Para-Athletes was related to people's experience of watching the Para-Sports and interest in the Paralympics. Therefore, it is important for the promotion activities to highlight this positive image.

This study developed questionnaire items based on the free-descriptive answers to the preliminary survey in order to investigate people's impressions. Some of the participants did not take part in both the preliminary and main surveys. There is room for improvement of the questionnaire items and their validity and reliability in the survey. The results of reliability analysis showed the questions had acceptable reliability, however, their validity was not verified in this study. Therefore, it is necessary to conduct surveys examining the relationship between the questionnaire in this study and other related questionnaires or participants' attributes for verification of validity. A future issue is to develop a more effective questionnaire, and further surveys are required.

\section{Conflicts of Interest}

The authors declare no conflicts of interest regarding the publication of this paper.

\section{References}

Fujita, M. (2016). A Study on Attitudes toward Sports for People with Disabilities, Paralympics, and People with Disabilities. Society of Health and Sports Science, No. 8, 1-13. (In Japanese)

Panasonic Co., Ltd. (2016). A Survey on Watching the 2016 Rio Olympic Games. Panasonic Newsroom Japan. (In Japanese)

https://news.panasonic.com/jp/topics/146355.html

Sato, H. (2015). The General Public's Awareness and Interest in the Paralympics in Japan and in Some Selected Countries. Paralympic Research Group Journal, 1, 63-71.

Yamaha Motor Foundation for Sports (2016). Chapter 1: The Current Situation and Issues of Disabled Sports. A Report of Basic Research and Study for Model Construction of Picking-Out and Development System for Athletes with Disabilities. (In Japanese) https://www.ymfs.jp/project/culture/survey/006/pdf/ymfs-report 20160406-3.pdf 